\title{
Achille-Cléophas Flaubert et les métamorphoses du regard chirurgical
}

Julie Aucagne

\section{(2) OpenEdition}

\section{Journals}

Édition électronique

URL : https://journals.openedition.org/recherchestravaux/238

DOI : 10.4000/recherchestravaux.238

ISSN : 1969-6434

Éditeur

UGA Éditions/Université Grenoble Alpes

\section{Édition imprimée}

Date de publication : 15 octobre 2007

Pagination : 125-137

ISBN : 978-2-84310-111-3

ISSN : 0151-1874

Référence électronique

Julie Aucagne, "Achille-Cléophas Flaubert et les métamorphoses du regard chirurgical », Recherches \& Travaux [En ligne], 71 | 2007, mis en ligne le 15 avril 2009, consulté le 21 septembre 2021. URL : http:// journals.openedition.org/recherchestravaux/238; DOI : https://doi.org/10.4000/recherchestravaux. 238 
Julie Aucagne

Université de Bourgogne

\section{Achille-Cléophas Flaubert et les métamorphoses du regard chirurgical}

L'image du « regard chirurgical » est récurrente dans L'Idiot de la famille: elle revient régulièrement scander les différents épisodes de la vie de Flaubert, avec la constance d'un thème musical, voire d'une obsession, mais aussi avec une remarquable faculté d'ubiquité. Au fil des pages on la voit circuler, disparaittre, resurgir ; le possesseur de ce regard est tour à tour un imposteur, un médiocre ou le détenteur d'une formidable et dangereuse puissance. L'expression recouvre en effet des réalités diverses : c'est d'abord un des poncifs de l'art poétique flaubertien, celui qui a contribué à inscrire durablement celui-ci, au regard de l'histoire littéraire - tout au moins dans ses versions scolaires -, dans la catégorie des « réalistes »; or Sartre, lecteur plus subtil, sent bien que chez Flaubert, le réaliste n'est que l'envers de l'artiste, l'homme imaginaire qui veut priver le monde de sa réalité en l'enfermant tout entier dans un livre. D'où vient cette tension entre les deux Flaubert, et dans quelle profondeur plonget-elle ses racines? Sur ce point l'avantage est que le regard chirurgical, censé symboliser la fameuse observation réaliste, renvoie aussi directement à l'histoire familiale et à la profession d'Achille-Cléophas Flaubert. Les biographes antérieurs ont voulu y voir, tantôt un hommage rendu à la science d'un père admiré, tantôt une tentative pour entrer en concurrence avec lui : fils de chirurgien, Flaubert se serait voulu chirurgien des lettres, en une sorte de saine émulation, la littérature s'appuyant sur le bras de la science ; mais Sartre soupçonne ce cliché critique et biographique de posséder une origine plus littérale. À ses yeux, le regard chirurgical est loin de n'être qu'une métaphore et, quand Flaubert, dans sa correspondance, l'emprunte à son père défunt, ce n'est pas un hommage : c'est une vengeance. De ce regard le fils n'est pas l'héritier mais la victime : comme Caïn, Gustave est poursuivi par l'œil implacable du Père. 
C'est pourquoi Achille-Cléophas, ce parangon de la bourgeoisie libérale, ne tarde pas à acquérir dans L'Idiot de la famille une stature mythique : derrière le voltairien réputé pour sa finesse d'esprit, le bon docteur respecté pour la sûreté de son diagnostic, Sartre débusquera en effet le père tyrannique et le disséqueur acharné de cadavres humains.

Rappelons d'abord les grandes étapes du processus qui, dans l'œuvre, permet la transmission du regard chirurgical du père au fils : elles permettent de voir comment Sartre remonte de la métaphore apparente à la réalité concrète qui l'a engendrée, et comment l'image fonctionne comme principe structurant de la figure flaubertienne. Tout commence par la disgrâce dont est victime le jeune Gustave, à l'âge de raison : le père, déçu par son cadet, se met à repousser ses manifestations de tendresse et met en cause leur sincérité ; or Gustave, "être-relatif », ne tire son être que du lien féodal qui l'attache à ce père adoré ; d'autre part, en tant qu'il est " passivement constitué », il ne peut bel et bien ressentir aucune émotion réelle, aucun désir, à moins qu'un regard autre ne lui confirme leur réalité : il lui faut donc les jouer, les offrir sans cesse à ce regard ; mais depuis cette " Chute $^{\mathrm{I}}$ », la nature de ce regard a changé. Achille-Cléophas refuse désormais d'être complice de cette « montre» (IDF, t. I, p. 669) : au contraire, son coup d'œil infaillible met à nu l'insincérité de son fils. Or « en déréalisant les conduites de l'enfant, [ses regards] [le font] pour toujours imposteur, c'est-à-dire autre que ce qu'il prétend être, sans lui dévoiler pour autant ce qu'il est » (IDF, t. I, p. 683) ; en effet, AchilleCléophas détient en outre aux yeux de son fils la vérité de la vie humaine; son commerce avec les cadavres la lui a révélée comme néant et comme pourriture ; par extension, son regard devient l'attribut du démystificateur, qui débusque chez les autres les mensonges et les faux-semblants : et le premier de ces mensonges, c'est la «bêtise de première instance ", autrement dit la «pensée théologique» (IDF, t. I, p. 642) qui fait prendre pour des manifestations de la vérité ces impostures : les cérémonies religieuses, les extases poétiques... mais aussi la vassalité du fils. Achille-Cléophas, le savant, devient alors le Démoralisateur satanique, celui qui retire du monde toute poésie.

La revanche viendra, mais elle attendra la seconde Chute, celle de PontL'Evêque. Gustave à cette occasion fait coup double : la maladie permet que lui soit délivré, "par une sommité médicale, un certificat d'incapacité à vivre » (IDF, t. II, p. I 889); mais tandis qu'il feint de se soumettre aux décrets du médecin, il accomplit en même temps, clandestinement, un parricide : l’impuissance de son père à le guérir le dénonce comme un incompétent en

I. Jean-Paul Sartre, L'Idiot de la famille, Gallimard, "Bibliothèque de philosophie ", t. I, p. 361. Toutes les références suivantes renvoient à cette édition, en trois volumes (I 97 I, rééd. I 988 pour les deux premiers; I972, rééd. I 988 pour le dernier). 
même temps que comme le coupable de sa maladie. Disqualifié comme père et comme savant, il n'aura plus qu'à mourir pour que son fils devienne enfin écrivain, détenteur à son tour de ce « regard chirurgical » qui s'est retourné contre son premier possesseur. Mais cet instrument de pouvoir, entre les mains - si l'on peut dire - de Gustave, restera problématique : ce sera toujours en lui une instance étrangère; il en sera hanté : il ne se l'appropriera pas.

La mécanique de ce roman familial est diabolique ; elle appelle plusieurs remarques : d'abord, ce « regard chirurgical» semble être le dernier avatar de l'« être-vu-par-derrière ${ }^{2}$ » qui traverse, pétrifie ou dissout nombre de personnages sartriens, de l'enfant des Mots sous le regard d'un grand-père comparé, comme Achille-Cléophas, à Moïse’ à Genet - tous voués par un regard qui s'incorpore à leur chair et installe, en eux, l'Autre. Le regard de ces Pères mauvais fige les fils dans l'impuissance, les aliène à une parole autoritaire qui, feignant de porter un diagnostic sur l'individu qu'elle perce à jour et définit - « tu es un idiot », " tu es un voleur »- ne fait que renvoyer ces prétendus connaisseurs du cœur humain à leurs propres insuffisances. Ainsi Achille-Cléophas n'est-il le détenteur que d'un savoir dévalué, qui met en jeu le corps, et non l'esprit: Sartre affirme qu'il sonde «les reins, non les cœurs »; et c'est ce rôle médiocre qu'il va lui faire jouer à l'heure de la déconfiture, pendant la maladie de son fils, après la crise de Pont-L'Evêque, cette «mortpar lapensée» (IDF, t. II, p. I799) : «Du matin au soir, entrant à l’improviste, il dévisage son fils, prend son pouls, fait tomber sur lui ce "regard chirurgical" qui détecte tous les mensonges. [...] il veut comprendre.» (IDF, t. II, p. I 89 I) Or, Achille-Cléophas ne comprend pas et «[se déconsidère] pour toujours » (IDF, t. II, p. I 890) : c'est la victoire de Flaubert, enfin devenu artiste en sa névrose, sur une autre imposture : la raison analytique, cette « connaissance en extériorité ou connaissance parcellaire» (IDF, t. I, p. I 265). De même, Canivet, autopsiant Charles Bovary, ne trouvera « rien ${ }^{4} »$ : Sartre semble reprendre à son compte l'ironie flaubertienne et affirmer, à mots couverts, que la vérité appartient au livre et pas à la dissection - à la totalisation contre le regard chirurgical.

\section{Achille-Cléophas, repoussoir et double de Sartre biographe}

Car Achille-Cléophas, c'est l'analyse en personne : à travers lui Sartre va instruire, non seulement le procès du scientisme balbutiant des années 1830, mais

2. L'expression est de Josette Pacaly, dans Sartre au miroir: une lecture psychanalytique de ses écrits biographiques, Klincksieck, 1985 , p. 246.

3. Les Mots, Gallimard, «Folio », I 964, p. I 30 . Voir aussi p. 6o, 85 et I 49.

4. Madame Bovary, Jean Pommier et Gabrielle Leuleu éd., José Corti, I949, p. 672. 
celui d'un mode de connaissance fondé sur l'observation et sur la dissection (souvent symbolique, mais pas toujours) des cadavres - science mortifère, à la fois passive (parce qu'elle est fondée sur l'observation et non sur l'action) et dotée d'une puissance d'aliénation surprenante. Il n'est donc pas étonnant que - bien que dans sa correspondance Flaubert emploie aussi volontiers l'expression de « coup d'œil médical » pour définir son art - Sartre ait préféré la « chirurgie » à la « médecine » : celle-ci guérit, recompose, apaise ; entre les mains de celle-là tout vivant est un mort en puissance, un corps livré, grotesque, que l'on ouvre, manipule, découpe tout à loisir - une série de pièces détachées de la machine humaine. Il s'agit donc d'abord pour Sartre de faire du médecin chef de l'Hôtel-Dieu le symbole d'une science mauvaise, dévoyée ; la science de i 830 , écrit-il, est « une science passive, qui analyse et ne recompose pas, $[\ldots]$ une science impuissante qui veut connaitre et ne sait pas guérir »(IDF, t. I, p. 472). Ainsi, dit encore Sartre, "Achille-Cléophas observe. Il dissèque avec zèle mais la dissection n'est souvent qu'un inventaire : on établit la carte géographique du corps humain ; on dresse, après décès, le procès-verbal des modifications que lui apporte la maladie » (IDF, t. I, p. 470). Se dessinent alors deux réseaux d'images - l'un explicite, qui renvoie à un mode de connaissance qui atomise le sujet et le réifie, l'autre sousentendu, qui lui oppose l'épistémologie sartrienne, où la totalisation vient sans cesse corriger les effets délétères de l'analyse, où le cadavre, objet déplié « sur la table de marbre » (IDF, t. I, p. 472) de l'amphithéâtre, est rendu à sa condition de sujet.

Ce qui est en jeu, ce n'est rien de moins que la guérison du mort. Savant et créateur paradoxal, Achille-Cléophas devant le corps découpé se retrouve alors dans la position du biographe devant ses matériaux épars, face à un cadavre en attente de résurrection; et si la comparaison s'impose, c'est qu'elle est loin d'être sans précédent dans l'histoire de la biographie. On la trouve notamment sous la plume d'un des plus illustres représentants du genre, Sainte-Beuve, qui définissait sa pratique comme une tentative de "glisser le scalpel et d'indiquer les défauts de la cuirasse, $[\ldots]$ de montrer les points de suture entre le talent et l'âmes ». Le même avouera que « la critique d'un écrivain sous notre plume court toujours le risque de devenir une légère dissection anatomique ${ }^{6} »$. Or ce qui sera reproché au "prince des critiques », c'est de dissimuler derrière cette métaphore agressive, invasive, une connaissance

5. Sainte-Beuve, Lettre à Ernest Bersot du 9 mai 1963, Correspondance générale, vol. I 3 , p. I 52. Cité par Gérald Antoine dans l'« Introduction » aux Portraits littéraires, Robert Laffont, «Bouquins", I993, p. LX.

6. Portraits contemporains, Calmann-Lévy, I 889, t. II, p. 35. 
superficielle et réductrice de ses modèles - et c'est pour cette raison même qu'Achille-Cléophas subit d'abord les foudres de Sartre :

Le docteur Flaubert ne se contente pas de fouiller les charognes : il est « comme un vieux prêtre chargé de secrets domestiques ». Des anecdotes, en somme : Achille-Cléophas prenait cela pour une « expérience du cœur humain » due bien sûr à l'art d'analyser les mensonges. [...] Gustave est ébloui : tous ces corps, tous ces cœurs « réduits en tronçons »! Le père devait, dans ces moments de faux abandon familial où le chef de famille pose devant sa femme et ses enfants, laisser entendre que les âmes aussi se dissèquent. Cette naïveté ne serait pas digne de remarque si elle n'avait fait tant de mal à son fils. (IDF, t. I, p. 488)

En effet la dissection ne fait pas seulement l'objet d'une réprobation intellectuelle : c'est elle qui pulvérise le sujet unitaire en « anecdotes », elle qui partant compromet sa résurrection - et qui vient symboliser les limites d'un genre douteux, trop confiant dans ses propres pouvoirs, et dont la soif de connaissance mal dirigée provoque paradoxalement la délitescence du sujet. C'est pourquoi quand Sartre place au cœur du texte de L'Idiot de la famille cette autre figure de savant, c'est pour la faire fonctionner comme avertissement, comme épouvantail régulièrement brandi pour tenter de tenir à distance le spectre d'une connaissance de l'autre qui serait émiettée, incomplète, aliénante voire morbide. Flaubert père ne serait donc pas seulement l'autre de Gustave, mais l'autre de Sartre biographe, penché comme lui sur un corps démembré qu'il convient de recoudre, et venant incarner un problème crucial : celui de l'altérité qui fonde la relation biographique.

Arrêtons-nous d'abord sur l'image récurrente du corps " tronçonné ». Cette image engendrera dans L'Idiot de la famille un Gustave "percé à jour ", dont les «mensonges » sont « désarticulés » et à qui finalement « la voie royale de la synthèse progressive » (IDF, t. I, p. 495) sera interdite. Sartre l'a relevée dans le passage de Madame Bovary où Flaubert fait le portrait du docteur Larivière, et plus précisément dans un membre de phrase supprimé de la version définitive. Quand le texte édité dit seulement : «Son regard, plus tranchant que ses bistouris, vous descendait droit dans l'âme et désarticulait tout mensonge à travers les allégations et les pudeurs », la version antérieure ajoutait : « et laissait tomber les tronçons à vos pieds ${ }^{7}$ ». Or, dit Sartre, « quand on veut comprendre les intentions de Gustave [...] l'important est moins ce qu'il publie que ce qu'il retire de son texte avant de le publier » (IDF, t. I, p. 457) : c'est donc la lecture entre les lignes des quelques pages concernant le docteur Larivière qui va transformer ce que les biographes antérieurs de Flaubert considéraient comme un hommage de Gustave à Achille-Cléophas en portrait à charge, et faire du savant magnifique « un vieux polichinelle» (IDF, t. I, 
p. 457); or, s'il est vrai que le portrait de Larivière n'est pas dénué d'ambiguité, il n'en reste pas moins que la lecture sartrienne est biaisée : c'est là qu'il faut appliquer au biographe sa propre méthode et considérer que, dans cette lecture, l'important n'est pas ce dont il parle, mais ce dont il omet de parler.

D’abord, affirme Sartre, il n'est pas vrai que le docteur Flaubert ait été bon; et même, la « débonnaireté » du docteur Larivière serait le contraire de la bonté ; pourquoi sinon le comparerait-on ailleurs à un « démon » ? Pourquoi soulignerait-on son manque de « complaisance » à l'égard des villageois ? La conclusion est implacable : Achille-Cléophas-Larivière est un faux vertueux (voir IDF, t. I, p. 454-459). Il « se donne les gants parfois, de soigner les pauvres pour rien » (IDF, t. I, p. 478) mais ne saurait comprendre ce que, par ailleurs, il connaît. En filigrane, c'est le modèle de l'empathie qui conteste cette connaissance altière : ainsi Achille-Cléophas, comme le docteur Mathurin dont il est le modèle, aura « la connaissance du mal mais non le mal de vivre » (IDF, t. I, p. 467); « il connaît [I'] horreur, scientifiquement, mais ne la ressent pas » (IDF, t. I, p. 457).

Ces efforts pour faire d'Achille-Cléophas un modèle négatif de science glacée seraient fort convaincants si l'on ne trouvait, dans les pages citées pour preuve, ce passage : «Bovary observa [le docteur] : ils se regardèrent; et cet homme, si habitué pourtant à l'aspect des douleurs, ne put retenir une larme qui tomba sur son jabot ${ }^{8} \gg \ldots$ Est-ce là un homme incapable de compassion ? À cette première distorsion du texte s'en ajoute une autre, qui a trait aux mains de Larivière, symbole de sa possibilité d'agir, de guérir, de sortir du domaine du constat - l'homme est un cadavre en puissance - pour changer le cours des choses. Sartre écrit de Flaubert :

[...] jamais nous ne le verrons envisager la médecine comme une lutte pour la vie; il la tient pour une science plutôt que pour un art. Du chirurgien, c'est le regard qu'il admire et non les mains. De son père, de Larivière, il feint de vanter le savoir théorique, les vertus mais non les guérisons qu'ils ont faites. (IDF, t. I, p. 473)

Or reprenons le portrait de Larivière : Flaubert s'y attarde au contraire à décrire «ses mains charnues, de fort belles mains, et qui n'avaient jamais de gants comme pour être plus promptes à plonger dans les misères9 »; et à

8. Ibid., p. 606.

9. Ce passage est extrait d'un paragraphe qui ne figure pas dans l'édition Pommier-Leuleu, où Sartre a puisé l'image du tronçonnement; il est cependant conservé dans plusieurs autres éditions (voir par exemple Madame Bovary, Euvres complètes de Gustave Flaubert, t. I, Club de l'honnête homme, I97 I, p. 332), et il est certain que Sartre le connaissait, puisqu'il le cite dans L'Idiot de la famille, t. I, p. I 25 , note $\mathrm{i}$. Or la relégation à la périphérie du texte de ce fragment souligne l'ambiguiité de la figure d'Achille-Cléophas en la révélant dans sa dimension proprement inintégrable à l'économie de la biographie : tout en constituant un point nodal de la synthèse, elle continue à la contester sourdement, à la menacer de son pouvoir d'atomisation. 
Homais qui lui dit: «J'ai voulu, docteur, tenter une analyse, et primo, j’ai délicatement introduit dans un tube... », Larivière réplique : « Il aurait mieux valu $[\ldots]$ lui introduire vos doigts dans la gorge ${ }^{10} »$; celui, qui, ici, répugne à « plonger les mains dans les misères » et se réfugie dans l'« analyse », gratuite et inefficace, c'est le pharmacien, non le chirurgien ; un détail encore : on aura peut-être noté que Sartre prétend qu'Achille-Cléophas ne faisait que «se donner les gants » de pratiquer la vertu (IDF, t. I, p. 478); or ces « gants » qui s'interposent entre l'homme de l'art et le corps souffrant, qui viennent nier toute possibilité de continuité entre l'observation du cadavre et l'action qui lui redonnerait vie - qui, en somme, dénoncent doublement l'imposture du savant - trouvent, il me semble, une résonance assez curieuse dans un passage des Mots. Sartre y parle de sa familiarité avec les grands écrivains, comme Baudelaire et, bien sûr, Flaubert, et des reproches qu'on lui adresse quant à la liberté avec laquelle il en parle. Il affirme alors qu'il a envie de répondre : « $\mathrm{Ne}$ vous mêlez pas de nos affaires. Ils m’ont appartenu, vos génies, et je les ai tenus dans mes mains, aimés à la passion, en toute irrévérence. Vais-je prendre des gants avec eux ${ }^{11}$ ? » En effet, "prendre des gants », c'est bon pour les Achille-Cléophas... mais ce qui est troublant ici, c'est que ce contact immédiat avec les génies s'associe dans l'autobiographie à des images de manipulation, de possession : le livre, ce « petit cercueil » entre les mains de l'enfant Sartre, se met à ressembler aux cadavres couchés sur les tables de l'amphithéâtre, voire à Gustave subissant, au berceau puis sur son lit de malade, les soins de ses parents. Entre le biographe-lecteur et le médecin-disséqueur, se dessine, au-delà de leur opposition apparente, une inquiétante parenté : l'effort pour briser le miroir laisse constamment apparaître son envers, la subsistance du reflet accusateur du tronçonneur, du démystificateur, du bourreau de Gustave.

L'effroi que suscite chez Sartre le soupçon de cette présence de l'Autre en soi explique peut-être que l'on ne cesse de glisser, dans L'Idiot de la famille, de la métaphore à la lettre. Le « regard chirurgical» ne se contente pas de figurer un modèle épistémologique révoqué avec dédain : l'analyse est bel et bien une forme de vivissection. Car « ce que [Gustave] reproche au Géniteur» à travers Madame Bovary, selon Sartre,

c'est d'avoir [...] lu à livre ouvert dans l'âme de son fils. Les rêves, les songes et les mensonges que son regard a violés, désarticulés, ce sont ceux de Gustave. Celuici, plus d'une fois, a vu sa vie profonde «tomber à ses pieds en tronçons ». (IDF, t. I, p. 459) 
Ce n'est donc plus d'un savant impuissant qu'il s'agit, mais d'un tortionnaire, d'un père épouvantable dont le regard inscrit dans la chaire vive de son cadet une mort effroyable. Gustave, sous l'œil inquisiteur d'Achille-Cléophas, dont le rire et le regard à chaque instant taillent dans le vif, l'écartèlent, ne peut que survivre d'une vie artificielle et toujours douteuse. Il n'est en somme qu'un de ces «morts ensorcelés », que l'auteur de ses jours crée à volonté pour satisfaire une infernale fringale épistémologique : en effet, « le pater familias n'aurait-il pas créé Gustave pour observer, vivant, son pourrissement et, mort, pour le disséquer tout à l'aise ?» (IDF, t. I, p. 475-476).

Or, si Achille-Cléophas voyait en son fils vivant un cadavre démembré, disponible pour l'analyse, Sartre voudrait faire un vivant de Flaubert mort. Écrire la vie de Gustave, c'est donc aussi réparer le corps du génie mutilé, défaire l'œuvre diabolique du père - se faire médecin en somme, et, en se substituant à ce premier " auteur » défaillant, recoudre les fragments d'une expérience vitale qui ne se transmet à la postérité que sous la forme de pièces détachées. Ce qui se dessine à travers la figure d'Achille-Cléophas, c'est donc la hantise d'une pratique biographique qui « taille » et ne recoud pas (IDF, t. I, p. 490), ne laissant subsister du mort que des "tronçons » épars entre lesquels se creusent d'insupportables béances. En Sartre, c'est le lecteur qui va tenter de conjurer l'éparpillement du sens et, en suturant ses plaies ouvertes, de réunifier l'objet Flaubert pour garantir son retour à la vie - au risque de se laisser tenter par l'acharnement thérapeutique, comme le suggère ironiquement Julian Barnes évoquant le devenir posthume des écrivains dans Flaubert's Parrot:

Regardez ce qui est arrivé à Flaubert : un siècle après sa mort, Sartre, comme un sauveteur costaud et désespéré, a passé dix ans à lui taper la poitrine et à lui souffler dans la bouche; dix ans à essayer de le ramener à la conscience, simplement pour pouvoir l'asseoir sur la plage et lui dire exactement ce qu'il pensait de lui ${ }^{12}$.

\section{Suturer, pontifer}

Les blessures de Flaubert ne sont nulle part aussi visibles que dans son œuvre. L'on a vu que la lecture du portrait de Larivière était entièrement fondée sur une phrase supprimée de Madame Bovary : or si cette pratique de la lecture en marge est une constante dans la biographie sartrienne, c'est que le

I 2. "Look what happened to Flaubert: a century after his death Sartre, like some brawny, desperate lifeguard, spent ten years beating on his chest and blowing into his mouth; ten years trying to yank bim back to consciousness, just so that he could sit him up on the sands and tell him exactly what he thought of him." Flaubert's Parrot, Londres, Picador, I984, p. 95. Nous traduisons. 
corpus flaubertien y est considéré comme un contingent d'énigmes - comme autant de fragments qui tentent de faire signe vers une totalité perdue, dont la vérité se serait réfugiée tout entière dans les silences. La lecture entre les lignes rachète le calvaire d'un Gustave démembré par le regard chirurgical, recoud les morceaux dispersés par le scalpel effroyable du père. Un des meilleurs exemples de cette pratique de la lecture comme suture est fourni par l'étude que fait Sartre de Novembre, l'œuvre écartelée de Flaubert : elle est en effet divisée en deux parties par ce que Sartre appelle une « coupure saignante » (IDF, t. II, p. I7I 2) ; dans l'une, le narrateur raconte, à la première personne, ses tourments et ses déboires; dans l'autre, un second narrateur évoque, après la mort du premier, ses derniers jours. D'où vient, demande Sartre, que Gustave se soit ainsi dédoublé, qu'il se situe d'abord en compréhension avec soimême, puis se prenne pour objet de connaissance ? La réponse est théâtralisée avec soin : «à la fin d'août ou au début de septembre, Gustave a voulu se tuer» (IDF, t. II, p. I72 I); il a alors connu, précise son biographe, une «crise d'estrangement » qui lui permet, pour une fois, de se percevoir du point de vue d'autrui, de réaliser que «le fils Flaubert, enfant imaginaire aux aspirations infinies, est en réalité un raté » (IDF, t. II, p. I723); c'est, conclut Sartre, le triomphe du « regard-autre» en Flaubert (IDF, t. II, p. 1724).

Nous savons déjà à qui appartient ce regard qui coupe en deux, et l'œuvre, et le jeune candidat à l'imaginaire ; on le reconnaît d'autant mieux, derrière la permanence des métaphores, que le second narrateur est décrit comme «posthume» (IDF, t. II, p. I720) : c'est le fait qu'il se situe du point de vue de la mort - la seule posture qui puisse permettre l'accès à « la vérité de la vie », aussi bien pour Sartre que pour Achille-Cléophas ${ }^{13}$ - qui va lui conférer son statut de démystificateur du premier. Lui aussi vient travailler sur le cadavre. Que trouve-t-on dans le gouffre silencieux et effroyable qui traverse l'œuvre? La maladie et le meurtre, dit Sartre ; autant dire Achille-Cléophas lui-même c'est-à-dire l'innommable de la névrose, « la face autre et le nom caché du mal qui n'a pas de nom» (IDF, t. I, p. 464). Ainsi la reconstitution des parties manquantes de l'œuvre s'apparente-t-elle à un exorcisme : il faut, à la lettre, faire taire l'innommable - précisément en le nommant. Sartre l'explique ailleurs : nommer ce qui n'est qu'obscurément vécu, c'est, en le projetant à l'extérieur, le transformer en objet (IDF, t. I, p. 784). À ce prix Achille-Cléophas cessera peut-être de hanter Gustave : en revanche, il se met à hanter, de manière lancinante, le discours sartrien.

I 3. Voir «Sur L'Idiot de la famille », dans Sartre, Situations X, Gallimard, I976, p. I05, et $I D F$, t. I, p. 490. 
En effet, cette tentative pour combler le vide qui se creuse entre les deux parties de Novembre s'accompagne d'une collusion d'images pour le moins troublante :

[...] le biographe [i.e. le second narrateur] se peint par son entreprise : ses propos dépouillés trahissent le souci constant de refuser le pathos. Mais, sous l'affectation d'impartialité, sous l'ironie condescendante, on découvre de la violence, un rythme étrange, heurté, précipité. Cet homme n'est pas détaché : nous le devinons sinistre, angoissé. En vérité nous ne pouvons le tenir pour le simple produit passif de la mort du héros : on l'a convoqué, dirait-on, dans un cas d'extrême urgence pour lui demander un diagnostic. De la vient, en partie, son attitude gourmée : c'est un médecin, ou un flic. S'il fait l'impartial, s'il bloque ses sentiments, c'est qu'il enquête : cherchant à comprendre les mobiles de l'accusé ou du patient, il s'interdit d'abord de les partager. Mais de quoi cherche-t-il les indices ou les symptômes ? D'un meurtre, ou d'une maladie ? C'est tout un. (IDF, t. I, p. I72 I)

Il s'agit bien ici de médecine, non de chirurgie ; quant au « flic », c'est probablement aussi celui du roman policier - celui qui reconstituera, au-delà du disparate des indices réunis par l'enquêteur, le récit continu du crime. Mais que les deux soient associés, et émerge la figure du légiste : revoici donc, sous les traits du biographe, Achille-Cléophas ; quant à la pratique de l'ironie, de la démystification systématique, à qui appartient-elle au juste ? au second narrateur de Novembre? aux Flaubert père et fils ? ou plutôt à Sartre biographe, traquant la preuve, proférant des diagnostics péremptoires et " [établissant] si Gustave est sincère » (IDF, t. I, p. 7) ? Encore une fois, nous voici confrontés à un phénomène d'inquiétante familiarité : la superposition des fonctions suggère une identification, au moins temporaire, à la figure de Flaubert le père signe, peut-être, d'une mauvaise conscience mêlée de fascination pour le chirurgien-repoussoir. Curieux paradoxe : la tentative de résurrection que constitue la reconstitution d'une partie manquante de l'œuvre continue à achopper sur la figure d'Achille-Cléophas et sur la double menace qu'il incarne - la fragmentation, et l'arbitraire. Or cette ambivalence qui marque la lecture-suture apparaissait déjà dans la pratique de l'enfant des Mots :

Je ne savais pas encore tronçonner les morts mais je leur imposais mes caprices : je les prenais dans mes bras, je les portais, je les déposais sur le parquet, je les ouvrais, je les refermais, je les tirais du néant pour les y replonger : c'étaient mes poupées, ces hommes-troncs, et j'avais pitié de cette misérable survie paralysée qu'on appelait leur immortalité ${ }^{14}$.

On conviendra que les activités de cet enfant compatissant rappellent une nouvelle fois de manière suspecte celles de Flaubert le père. «Tirer du néant » les morts implique-t-il forcément de les ouvrir et de les refermer comme le 
chirurgien-chef, avec application, décompose et recompose un cadavre qu'il oublie de rendre à la vie ? Et dépasser cette « misérable survie paralysée » de l'homme-tronc qu'est l'auteur post mortem, est-ce autre chose, finalement, que lui «imposer ses caprices »? C’est que, pour tirer de ce cadavre tronqué qu'est l'œuvre un auteur bien vivant, il ne suffit pas de recoudre : il faut recoudre bien, et ne pas oublier de galvaniser le mort ainsi reconstitué. Sartre biographe, comme avant lui Achille-Cléophas, ce « créateur de morts ensorcelés » (IDF, t. I, p. 490), est sans doute victime de ce mal courant chez ses confrères, et que j'appellerais volontiers complexe de Frankenstein : la peur d'être un créateur de monstres, d'êtres paradoxaux - morts vivants ou créatures composites - un nécromant indélicat qui animerait le mort d'une vie précaire, factice, étrangère. Or si la hantise de cette biographie dévoyée traverse tout L'Idiot de la famille, il faut se souvenir que le thème apparait aussi... chez Flaubert lui-même.

Il faut en effet se reporter à l'épisode de Bouvard et Pécuchet où les deux compères tentent d'écrire une vie du duc d'Angoulême : ils réunissent d'abord à cet effet une série d'informations - dates, hauts faits, anecdotes et «beaux mots »- et un portrait,

une lithographie coloriée, représentant de trois quarts, Monseigneur le duc d'Angoulême.

Le drap bleu de son habit d'uniforme disparaissait sous les épaulettes, les crachats, et le grand cordon rouge de la Légion d'honneur. Un collet extrêmement haut enfermait son long cou. Sa tête piriforme était encadrée par les frisons de sa chevelure et de ses minces favoris ; - et de lourdes paupières, un nez très fort et de grosses lèvres donnaient à sa figure une impression de bonté insignifiante ${ }^{15}$.

Les lacunes, la disparité et l'insignifiance de leurs sources sont manifestes, mais ne découragent pas les deux cloportes - en tout cas pas avant qu'un bibliothécaire trop zélé ne provoque, sans le vouloir, une catastrophe qui mettra fin à l'entreprise :

Au moment de partir, le bibliothécaire se ravisant, leur fit voir un autre portrait du duc d'Angoulême.

Sur celui-là, il était en colonel de cuirassiers, de profil, l'œil encore plus petit, la bouche ouverte, avec des cheveux plats, voltigeant.

Comment concilier les deux portraits? Avait-il les cheveux plats, ou bien crépus, à moins qu'il ne poussât la coquetterie jusqu'à se faire friser?

Question grave, selon Pécuchet; car la chevelure donne le tempérament, le tempérament de l'individu.

Bouvard pensait qu'on ne sait rien d'un homme tant qu'on ignore ses passions ${ }^{16}$.

I 5. Bowvard et Pécuchet, Euvres complètes de Flaubert, t. 5, Club de l'honnête homme, i972, p. I 3 I.

I6. Ibid. p. I 33 . 
Selon Françoise Gaillard, ce texte « mime la faillite d'une certaine conception de l'écriture, en l'occurrence son impuissance à résorber les sens parasitaires pour les soumettre à la loi du Tout, son incapacité à résoudre les problèmes des résidus, des surplus de signification ${ }^{17}$ »- soit le modèle incarné par Achille-Cléophas et révoqué par Sartre. Or la déroute de Bouvard et Pécuchet est d'autant plus déplorable qu'ils possédaient sans le savoir un remède à la dissolution du modèle biographique : ils avaient en effet repéré dans la vie du duc un motif récurrent, qui aurait pu fonctionner comme principe d'unification du disparate. Il apparait dans l'une de leurs notes :

On doit relever [dans la carrière du duc] l'importance qu'eurent les ponts. D'abord, il s'expose inutilement sur le pont de l'Inn, il enlève le Pont-Saint-Esprit et le pont de Lauriol; à Lyon, les deux ponts lui sont funestes - et sa fortune expire devant le pont de Sèvres ${ }^{18}$.

Or, comme le souligne Françoise Gaillard, le pont fonctionne ici comme «un truchement rhétorique»:

Ce pont qui, tel un gigantesque point de suture rapprochant les lèvres de la plaie du sens, jette son arche de chaque côté de la faille de l'histoire pour en souder artificiellement les deux bords, métaphorise ici la parole que Maurice Blanchot appelle «pontifiante », la parole bouche-trou, celle qui franchit allègrement l'abîme sans le combler, qui propose le nappé de son ordre pour pallier le chaos du monde, qui offre sa continuité fictive pour faire oublier la discontinuité originelle ${ }^{19}$.

La parole pontifiante, c'est donc cette «parole de maitrise ${ }^{20}$ » autoritaire qui conjure l'angoisse du retrait du sens et de la dislocation du sujet - celle que l'on prononce pour simuler la continuité de l'objet de la connaissance. Or il est certain que le biographe qui pontifie risque à tout moment l'abus de pouvoir - la trahison du modèle étant peut-être le prix de l'unité du biographé. En effet, si à l'horizon de la fréquentation des génies morts par le jeune Poulou, se profilait le risque de l'arbitraire et de son envers, le tronçonnement ( je ne savais pas encore tronçonner les morts »), celui-ci ne décrit rien d'autre ici que l'activité de professeur et de traducteur de Charles Schweitzer, débitant les classiques en extraits pour les changer en exercices de thème et de version. Il s'agit en somme pour lui de découper pour mieux pontifier - c'est-à-dire recréer un sens, annuler l'obscurité et l'étrangeté radicale du texte. Or ce mouvement qui fait suivre le tronçonnement d'une reprise du pouvoir - la création d'un

I7. Françoise Gaillard, «Une inénarrable histoire », Flaubert et le «comble de l'art »: nowvelles recherches sur Bouvard et Pécuchet, colloque des 22 et 23 mars I 980 , Société des études romantiques et Société d'édition d'enseignement supérieur, I98 I, p. 76.

I 8. Bouvard et Pécuchet, éd. citée, p. I 32.

iq. F. Gaillard, art. cité, p. 86.

20. Ibid. 
gouffre, puis son franchissement - est une constante de la rhétorique sartrienne, et fonde l'écriture de tout L'Idiot de la famille: ne s'agit-t-il pas de «comprendre ce scandale : un idiot qui devient génie »? Au commencement est la rupture - le «scandale » - : l'idiot et le génie, mais aussi le réaliste et l'artiste, la défaite et la victoire de Madame Bovary, etc., forment autant de couples antagonistes posés au seuil de l'œuvre, dont il faudra ensuite tisser ensemble les fils. Le tronçonnement est, comme chez Karl, le préalable à la récupération d'un sens qui induit cependant une distorsion de la parole originale.

Car pontifer, c'est aussi attester, pour reprendre l'expression de Françoise Gaillard, de sa «maittrise de la parole »- en même temps que sa propension à s'approprier la parole de l'autre. Pour le biographe Sartre, traduire l'œuvre de Flaubert en en évacuant les résistances au sens, " dire l'indisable », implique également de balayer d'un revers de la main la «tentative absurde » (IDF, t. III, p. 662) que serait Bouvard et Pécuchet - l'œuvre totale paradoxalement faite de pièces et de morceaux - pour y substituer les textes rafistolés de Novembre, Madame Bovary et tant d'autres : et nier ainsi le gouffre, n'est-ce pas aussi une façon, pour reprendre l'expression employée par Michel Sicard dans un entretien avec Sartre, de « corriger Flaubert »?

On a [...] l'impression que vous corrigez progressivement Flaubert, au double sens du mot : vous lui infligez des sévices et finalement vous réécrivez le texte avec tout ce qui manque (le fameux indisable) ${ }^{21}$ !

Ainsi Flaubert sera-t-il sauvé en dépit de lui-même : dans cette perspective, le « regard chirurgical » serait bien dans L'Idiot de la famille une parole «pontifiante »- parole qui saura recoudre les plaies de Flaubert, l'homme et l'œuvre, au prix d'un détournement : celui d'une parole flaubertienne disséquée, traduite et finalement volée par son biographe. Quant à Achille-Cléophas, à travers les métamorphoses de son « regard chirurgical», il semble bien s'imposer comme le support d'un métadiscours implicite qui souvent s'écrit sous la forme d'un psychodrame, parce que sa mise à distance n'est jamais garantie, jamais définitive - comme si les affres de Flaubert écrivain répondait à ceux de son biographe, hanté par la question de la connaissance de l'Autre, et de son retour incertain à la vie.

2 I. M. Sicard, «Sartre parle de Flaubert», Essais sur Sartre, Galilée, I 989, p. I66. 